\title{
Características clínicas y microbiológicas de la infección intra-abdominal complicada en Colombia: un estudio multicéntrico
}

\author{
Marta Vallejo, Diana P. Cuesta, Luz E. Flórez, Adriana Correa, Carmen E. Llanos, \\ Berenice Isaza, Stella Vanegas, Johanna Osorio, Lucía Casanova y María V. Villegas
}

\section{Clinical and microbiological characteristics of complicated intra-abdominal infection in Colombia: a multicenter study}

Introduction: Complicated community-acquired intra-abdominal infections (CA-cIAI) are a common cause of acute abdomen. Objective: To identify the clinical and microbiology profile of CA-cIAI in four Colombian hospitals. Methods: This is a prospective, descriptive study, between 08-2012 and 09-2014, including patients with CA-cIAI $\geq 15$ years. Data collected included: socio-demographic, clinical, diagnosis, and isolates of the first culture obtained aseptically during surgery with antimicrobial susceptibility. Results: 192 patients were included, $62 \%$ men, median age 47.3 years. Co-morbidities were present in $38.4 \%, 13 \%$ had been hospitalized in the previous year $13 \%$, and $9.4 \%$ had received antibiotics in the last 6 months; $44.3 \%$ were admitted for appendicitis, $17.7 \%$ for peritonitis and $16.7 \%$ for bowel perforation. CA-cIAI were assessed as moderate in $64.1 \%$ of the cases and were treated with ampicillin/sulbactam (SAM) and ertapenem. In $70.8 \%$ of cases a bacteria was isolated: $65.1 \%$ were gramnegative rods $(80.0 \%$ Escherichia coli, $44.8 \%$ of them susceptible to pipercillin/tazobactam, $65.7 \%$ to SAM; $11.2 \%$ were $K$. pneumoniae, $85 \%$ was susceptible for SAM; $16.7 \%$ were grampositive cocci $(28.1 \%$ Streptococci viridans group). The median hospital stay was 7 days and $15.1 \%$ died. Conclusions: E. coli, K. pneumoniae and $S$. viridans were the main organisms to consider in an empiric therapy for CA-cIAI and it is important to know the local epidemiology in order to choose the right antibiotic.

Key words: Peritonitis/microbiology; drug resistance, microbial; community-acquired infections, abdominal infection.

Palabras clave: Peritonitis/microbiología; fármaco-resistencia microbiana; infecciones comunitarias, infección abdominal.

\section{Introducción}

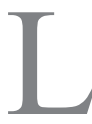

a infección intra-abdominal (IIA) es una de las causas más frecuentes de abdomen agudo que puede adquirirse en la comunidad (IIAc-AC) o durante la hospitalización (IIA-H). La primera presentación es más frecuente y representa $23 \%$ de las consultas médicas por dolor abdominal ${ }^{1}$. Según la localización de la infección, están involucradas distintas bacterias aerobias y anaerobias grampositivas y gramnegativas de la microbiota gastrointestinal del paciente. Los bacilos entéricos gramnegativos (Escherichia coli, Klebsiella sp), cocáceas grampositivas (Enterococcus sp) y microorganismos anaerobios estrictos (Bacteroides fragilis) son los patógenos predominantes, aunque la microbiología de la IIA varía según si su origen es comunitario o nosocomial ${ }^{2-3}$.

A partir de los reportes microbiológicos de cultivos positivos de pacientes con IIA, se han publicado diversos estudios de vigilancia de la microbiología y susceptibili- dad antimicrobiana de la IIA. En Latinoamérica, Villegas y cols., encontraron que 31,4 y $24,9 \%$ de los aislados en IIAc-AC e IIA-H, respectivamente, corresponden a microorganismos productores de $\beta$-lactamasas de espectro extendido (BLEE) con una susceptibilidad reducida a cefalosporinas de tercera y cuarta generación ${ }^{4-5}$. El reporte más reciente del programa de vigilancia de la IIA Study for Monitoring Antimicrobial Resistance Trends (SMART) describió en el período 2010-2011 que 54, 14, y 8\% de los aislados de IIAc-AC fueron E. coli, K. pneumoniae y Pseudomonas aeruginosa, respectivamente; adicionalmente se observó una susceptibilidad elevada para amikacina, ertapenem e imipenem, y disminuida para cefalosporinas y fluoroquinolonas ${ }^{6}$.

El manejo de la IIA complicada se realiza acorde a la gravedad de la infección e incluye la administración de líquidos de resucitación, el inicio de la terapia antimicrobiana una vez que se sospecha o confirma el diagnóstico de IIA, la realización de procedimientos de control del
Universidad Pontificia Bolivariana, Medellín, Colombia (MV, DPC). Hospital General de Medellín, Colombia (LEF). Centro Internacional de Entrenamiento e Investigaciones Médicas (CIDEIM), Cali, Colombia (AC, MVV).

Hospital Universitario San Jorge, Pereira, Colombia (CEL, BI).

Fundación Santa Fe de Bogotá, Bogotá, Colombia (SV). Hospital Universitario Hernando Moncaleano Perdomo, Neiva, Colombia (JO, LC).

Conflictos de intereses: Marta Vallejo ha recibido financiación para investigación de Merck Sharp \& Dohme, ha participado en comité consultor para AstraZeneca Colombia S.A., y ha sido conferencista de Merck Sharp \& Dohme, Merck Colombia, Pfizer Colombia, Abbott Colombia, bioMérieux Colombia S.A.S., Grupo Familia y Electroquímica West S.A. Diana Cuesta ha recibido financiación para investigación de Merck Sharp \& Dohme. María Virginia Villegas ha recibido financiación para investigación como miembro del Grupo de Resistencia Bacteriana Nosocomial en Colombia y ha sido conferencista y asesor de Merck Sharp \& Dohme, Merck Colombia, Novartis, Pfizer, AstraZeneca, Zambon, Abbott y Electroquímica West S.A. Los autores restantes declaran no tener conflicto de interés. Este estudio recibió soporte financiero para su implementación de Merck Sharp \& Dohme (contrato No 38344)

Recibido: 9 de abril de 2015 Aceptado: 17 de marzo de 2016

Correspondencia a: Marta Vallejo Agudelo vallejomarta@yahoo.com 
foco infeccioso, con obtención de cultivos microbiológicos según el riesgo del paciente y el conocimiento de la susceptibilidad del microorganismo más frecuentemente aislado de la IIA? .

El inicio del tratamiento antimicrobiano en IIAc-AC se hace empíricamente antes de que se conozcan los resultados del cultivo microbiológico, teniendo en cuenta si está o no localizado el foco infeccioso y si existe el riesgo de infección por patógenos resistentes ${ }^{8}$. El fracaso terapéutico empírico tiene consecuencias clínicas en los pacientes; se ha asociado a un mayor riesgo de complicaciones infecciosas, entre ellas la infección del sitio operatorio, y muerte ${ }^{9}$; a un aumento en 2,9 días de la duración de la terapia antibiótica parenteral (IC 95\% 0,8-4,9), 5,3 días adicionales de estancia (IC 95\% 2-8,5) y mayores costos de hospitalización que los pacientes con IIAc-AC con terapia empírica exitosa ${ }^{10}$.

El objetivo de este estudio observacional, multicéntrico, fue identificar el perfil clínico y microbiológico de la IIAc-AC en centros hospitalarios colombianos de mediana y alta complejidad, con el fin de brindar información para optimizar la selección de los antimicrobianos utilizados en la terapia empírica de pacientes con IIAc-AC y así minimizar las consecuencias negativas en la morbi-mortalidad de los pacientes y en los recursos institucionales.

\section{Pacientes y Métodos}

Se realizó un estudio descriptivo en pacientes con edad $\geq 15$ años en cuatro hospitales de mediana y alta complejidad de las ciudades colombianas de Medellín (Hospital General de Medellín), Neiva (Hospital Universitario Hernando Moncaleano Perdomo), Pereira (Hospital Universitario San Jorge) y Bogotá (Fundación Santa Fe), entre julio de 2012 y septiembre de 2014.

\section{Criterios de inclusión}

Se incluyeron, prospectivamente, a pacientes hasta completar el tamaño de la muestra, que cumplieran con:

- Presencia de una IIAc-AC, definida como una infección peritoneal con inflamación y/o perforación del tracto gastrointestinal o víscera hueca ${ }^{2}$, en un paciente con una internación menor a tres noches hospitalarias ${ }^{11}$,

- Indicación de tratamiento quirúrgico o de drenaje por métodos percutáneos guiado por imágenes, y

- Obtención de una muestra en quirófano para cultivo microbiológico del sitio de la infección, previa al inicio del tratamiento.

\section{Criterios de exclusión}

Se excluyeron aquellos con infecciones de origen ginecológico, ascitis, cirrosis hepática o con peritonitis hospitalaria, así como con información incompleta del reporte microbiológico de los aislados.
Cada investigador evaluó en su institución los criterios de selección de los pacientes, revisó la historia clínica y los reportes del primer cultivo microbiológico del sitio de infección. Se identificaron las variables edad, sexo, prescripción de antimicrobianos en los último seis meses y hospitalización previa durante el último año; enfermedades crónicas concomitantes que el paciente hubiera referido a su ingreso, diagnóstico de ingreso y clasificación de la IIA definida por el médico tratante; la estancia hospitalaria definida como días entre el ingreso a la institución y el egreso; estado vital al egreso. En caso de muerte, el médico tratante evaluó si fue atribuible a la infección y se indicó como tal en la historia clínica. Para este estudio, los investigadores clasificaron la IIA teniendo en cuenta la localización, y riesgo del paciente en: foco removible (infección sin perforación de víscera hueca, ni presencia de pus), infección leve (peritonitis localizada, sin signos de sepsis), infección moderada (peritonitis generalizada, con o sin signos y síntomas de infección sistémica leve o moderada y con co-morbilidades como diabetes mellitus, enfermedad pulmonar obstructiva crónica, insuficiencia renal) e infección grave (peritonitis generalizada, con signos sistémicos de infección y/o sepsis grave o shock séptico).

Cada hospital tenía incluido en el protocolo de manejo de los pacientes con IIA la obtención de un cultivo con técnica aséptica del sitio de infección durante el procedimiento quirúrgico o de drenaje, con el fin de identificar la bacteria causal y su perfil de susceptibilidad antimicrobiana.

Los procesos de toma de muestras, transporte al laboratorio clínico-microbiológico del hospital e identificación de la bacteria y susceptibilidad antimicrobiana por métodos automatizados, tipo VITEK ${ }^{\circledR} 2$ compack, fueron estandarizados en cada hospital por un investigador del estudio. No se realizaron procesamientos específicos para cultivar hongos y especies anaerobias estrictas, sí una estimación de su presencia con la tinción de Gram directa de la muestra.

Como control de calidad de los datos, un investigador revisó los formatos de recolección y verificó la información con la historia clínica y del reporte microbiológico.

Esta investigación obtuvo la aprobación del Comité de Ética de Investigación en Salud de la Universidad Pontificia Bolivariana y de las instituciones participantes.

\section{Análisis estadístico}

Para el estudio se estimó un tamaño de muestra de 185 pacientes con una precisión de $7 \%$, nivel de confianza de $95 \%$ y una frecuencia esperada de aislamiento de $E$. coli de $38,1 \%{ }^{3}$, muestra estimada con el software para estadísticas sobre epidemiología, OpenEpi.

Se presentó un análisis descriptivo de frecuencias para las variables socio-demográficas, prescripción de antimi- 
crobianos en los últimos seis meses, hospitalización previa durante el último año, enfermedades crónicas concomitantes, diagnóstico de ingreso, clasificación de la infección, fenotipo microbiológico y perfil de susceptibilidad in vitro a antimicrobianos. La distribución teórica normal de las variables cuantitativas fue evaluada con la prueba de Kolmogorv-Smirnov y aquellas con distribución normal se describieron con el promedio y desviación estándar, o mediana y rango intercuartílico (RIQ: percentil 25-percentil 75). No se realizaron comparaciones entre grupos.

\section{Resultados}

Se incluyeron 192 pacientes con IIAc-AC en el período de estudio con una edad promedio de 47,3 $\pm 20,6$ años; $62 \%$ eran de sexo masculino. El $38,4 \%$ padecía al menos una co-morbilidad al momento de la admisión hospitalaria siendo las más frecuentes: hipertensión arterial $(18,8 \%)$, condiciones de inmunosupresión $(7,8 \%)$ y diabetes mellitus $(5,7 \%)$ (Tabla 1$)$. Trece por ciento de los pacientes habían estado hospitalizados en el último año y $9,4 \%$ habían recibido antimicrobianos en los últimos seis meses.

Los diagnósticos de ingreso fueron: apendicitis $(44,3 \%)$, peritonitis $(17,7 \%)$, perforación intestinal (16,7\%) y absceso hepático (7,3\%), otros (14\%). La IIAcAC fue clasificada predominantemente como moderada en $64,1 \%$ de los pacientes, seguida por la infección leve en $22,4 \%$, grave $12 \%$ y $1,6 \%$ identificada como foco removible (Tabla 1$)$.

Los antimicrobianos que recibieron más frecuentemente los pacientes como tratamiento de la IIAc-AC fueron ampicilina/sulbactam 55,8\%, ertapenem $43,7 \%$, metronidazol $15,3 \%$ y meropenem $8,4 \%$ (Tabla 2). La mediana de la dosis correspondiente a ampicilina/sulbactam fue $12 \mathrm{~g} /$ día (RIQ: 9-12) con una mediana de duración de tres días (RIQ: 2-7). La dosis de ertapenem fue de $1 \mathrm{~g}$ /día con una mediana de duración seis días (RIQ: 3-6). Metronidazol y meropenem fueron prescritos a una dosis de 1,5 y $3 \mathrm{~g} /$ día y una mediana de duración de 7 días (RIQ: 3,3-9,8) y 9 días (RIQ: 4,5-16,3), respectivamente.

En 70,8\% (136/192) de los pacientes se aisló al menos un microorganismo; de ellos, $65,1 \%$ de los casos tuvo aislamiento de bacilos gramnegativos y $16,7 \%$ cocáceas grampositivas. De 135 pacientes en quienes se realizó el proceso de visualización de hongos y especies anaerobias estrictas, en $9(6,6 \%)$ y en $3(2,2 \%)$ se reportaron hongos y anaerobios estrictos, respectivamente; en 57 pacientes no se realizó esta búsqueda. Entre los bacilos gramnegativos aislados se encontraron $E$. coli $(80,0 \%), K$. pneumoniae $(11,2 \%)$, P. aeruginosa $(4,8 \%)$ y E. cloacae $(4,0 \%)$ como los prevalentes. Seis aislados de $E$. coli eran productoras de BLEE. Los aislados grampositivos más frecuentes fueron Streptococcus del grupo viridans $(28,1 \%), S$. anginosus y $S$. constellatus, ambos con $15,6 \%$ (Tabla 3 ).
Tabla 1. Características demográficas y clínicas de los pacientes con infección intra-abdominal adquirida en la comunidad en cuatro hospitales colombianos (n: 192)

\begin{tabular}{|c|c|}
\hline Característica & n (\%) \\
\hline Sexo masculino & $119(62,0)$ \\
\hline Edad años, media (desviación estándar) & $47,9(20,6)$ \\
\hline Hospitalización previa en último año & $25(14,0)^{*}$ \\
\hline Antimicrobianos últimos seis meses & $18(10,1)^{*}$ \\
\hline Co-morbilidad, al menos una & $73(38,0)$ \\
\hline Hipertensión arterial & $36(18,8)$ \\
\hline Inmunosupresión & $14(7,8)$ \\
\hline Enfermedad neoplásica & $12(6,3)$ \\
\hline Infección por VIH & $2(1,0)$ \\
\hline Uso de terapia biológica & $1(0,5)$ \\
\hline Uso de corticoesteroides & $0 \quad(0,0)$ \\
\hline Trasplante de órganos & $0 \quad(0,0)$ \\
\hline Diabetes mellitus & $10(5,7)$ \\
\hline Enfermedad renal & $9(4,7)$ \\
\hline Enfermedad respiratoria & $8(4,2)$ \\
\hline Falla cardiaca congestiva & $7(3,6)$ \\
\hline Enfermedad cerebrovascular & $0 \quad(0,0)$ \\
\hline Enfermedad hepática & $0 \quad(0,0)$ \\
\hline Otra enfermedad & $32(16,7)$ \\
\hline Farmacodependencia & $9(4,7)$ \\
\hline \multicolumn{2}{|l|}{ Diagnóstico al ingreso } \\
\hline Apendicitis & $85(44,3)$ \\
\hline Peritonitis & $34(17,7)$ \\
\hline Perforación intestinal & $32(16,7)$ \\
\hline Absceso hepático & $14(7,3)$ \\
\hline Perforación gástrica & $14(7,3)$ \\
\hline Úlcera duodenal perforada & $12(6,3)$ \\
\hline Colecistitis aguda & $9(4,7)$ \\
\hline Diverticulitis & $7(3,6)$ \\
\hline Absceso peritoneal & $6(3,1)$ \\
\hline Otro diagnóstico al ingreso & $17(8,9)$ \\
\hline \multicolumn{2}{|l|}{ Clasificación de la infección } \\
\hline Foco removible & $3(1,6)$ \\
\hline Infección leve & $43(22,4)$ \\
\hline Infección moderada & $123(64,1)$ \\
\hline Infección grave & $22(12,0)$ \\
\hline
\end{tabular}

La susceptibilidad antimicrobiana de los aislados de E. coli evaluados para piperacilina-tazobactam fue $44,8 \%$, ampicilina-sulbactam $65,7 \%$, gentamicina $81,6 \%$, ciprofloxacina $84,0 \%$, uno de tres aislamientos fue sensible a cefoxitina; para el resto de los antibióticos evaluados se evidencian unos perfiles de susceptibilidad superiores a $92 \%$. En los aislados de K. pneumoniae la susceptibilidad antimicrobiana reportada para ciprofloxacina y amikacina fue 13/14 (92,8\%), ampicilina/sulbactam 11/13 (84,6\%) (Tabla 4). 
Tabla 2. Tratamiento antimicrobiano en pacientes con infección intra-abdominal adquirida en la comunidad en cuatro hospitales colombianos ( $\mathrm{n}: 192)$

\begin{tabular}{lcc}
\hline Antimicrobianos recibidos & $\mathbf{n}$ & $(\%)$ \\
\hline Ampicilina/sulbactam & 106 & $(55,8)$ \\
\hline Ertapenem & 83 & $(43,7)$ \\
\hline Metronidazol & 29 & $(15,3)$ \\
\hline Meropenem & 16 & $(8,4)$ \\
\hline Vancomicina & 9 & $(4,7)$ \\
\hline Amikacina & 5 & $(2,6)$ \\
\hline Cefepime & 5 & $(2,6)$ \\
\hline Piperacilina/tazobactam & 5 & $(2,6)$ \\
\hline Ceftriaxona & 4 & $(2,1)$ \\
\hline Gentamicina & 4 & $(2,1)$ \\
\hline Doripenem & 3 & $(1,6)$ \\
\hline Ampicilina & 2 & $(1,1)$ \\
\hline Cefazolina & 2 & $(1,1)$ \\
\hline Ciprofloxacina & 2 & $(1,1)$ \\
\hline Clindamicina & 2 & $(1,1)$ \\
\hline Imipenem & 2 & $(1,1)$ \\
\hline Aztreonam & 1 & $(0,5)$ \\
\hline Colistina & 1 & $(0,5)$ \\
\hline Fluconazol & 1 & $(0,5)$ \\
\hline Norfloxacina & 1 & $(0,5)$ \\
\hline Linezolid & 1 & $(0,5)$ \\
\hline Oxacilina & 1 & $(0,5)$ \\
\hline & & \\
\hline
\end{tabular}

En los nueve aislados de $S$. del grupo viridans, 7/7 fueron sensibles a penicilina, $3 / 3$ a clindamicina y eritromicina y $2 / 2$ a ampicilina.

La mediana de estancia hospitalaria fue 7 días (P25P75: 5-11). Durante la hospitalización fallecieron 29 pacientes $(15,1 \%)$, y fue considerada como muerte atribuible a la IIAc-AC, según criterio del médico tratante, en $65,5 \%$ de ellos.

\section{Discusión}

La realización de este estudio multicéntrico en cuatro ciudades colombianas constituye el primero de nuestro país que identifica al paciente con IIAc-AC a partir del diagnóstico clínico de la infección y describe los resultados del cultivo microbiológico obtenido durante la cirugía, en contraste con otros estudios que seleccionaron al paciente a partir de los aislados positivos reportados en el laboratorio clínico.
Tabla 3. Hallazgos microbiológicos en pacientes con infección intra-abdominal adquirida en la comunidad en cuatro hospitales colombianos ( $\mathrm{n}: 192$ )

\begin{tabular}{|c|c|}
\hline Muestras & n $\quad(\%)$ \\
\hline Ningún aislamiento & $56(29,2)$ \\
\hline Al menos un aislamiento & $136(70,8)$ \\
\hline Especie única & $104(76,4)$ \\
\hline Múltiples especies & $32(23,6)$ \\
\hline Bacilos gramnegativos & $125(65,1)$ \\
\hline Escherichia coli & $100(80,0)$ \\
\hline Escherichia coli BLEE $(+)$ & $6(6,0)$ \\
\hline Klebsiella pneumoniae & $14(11,2)$ \\
\hline Klebsiella pneumoniae BLEE (+) & $0 \quad(0,0)$ \\
\hline Pseudomonas aeruginosa & $6(4,8)$ \\
\hline Enterobacter cloacae & $5(4,0)$ \\
\hline Citrobacter freundii & $2(1,6)$ \\
\hline Acinetobacter baumannii & $2(1,6)$ \\
\hline Enterobacter aerogenes & $1(0,8)$ \\
\hline Klebsiella oxytoca & $1(0,8)$ \\
\hline Aeromonas sobria & $1 \quad(0,8)$ \\
\hline Aeromonas hydrophila & $1(0,8)$ \\
\hline Hafnia alvei & $1(0,8)$ \\
\hline Proteus penneri & $1(0,8)$ \\
\hline Haemophilus parainfluenzae & $1(0,8)$ \\
\hline Enterobacter sp & $1(0,8)$ \\
\hline Salmonella sp & $1(0,8)$ \\
\hline Cocáceas grampositivas & $32(16,7)$ \\
\hline Streptococcus grupo viridans & $9(28,1)$ \\
\hline Streptococcus anginosus & $5(15,6)$ \\
\hline Streptococcus constellatus & $5(15,6)$ \\
\hline Streptococcus faecalis & $2(6,3)$ \\
\hline Enterococcus sp. & $2(6,3)$ \\
\hline Streptococcus infantarius & $1(3,1)$ \\
\hline Streptococcus faecium & $1(3,1)$ \\
\hline Streptococcus mitis & $1(3,1)$ \\
\hline Streptococcus ssp. & $1(3,1)$ \\
\hline Leuconostoc mesenteroides ssp cremoris & $1(3,1)$ \\
\hline Pediococcus pentosaceus & $1(3,1)$ \\
\hline Staphylococcus aureus & $1(3,1)$ \\
\hline Hongos & $9(6,6)^{*}$ \\
\hline Anaerobios estrictos & $3(2,2)^{*}$ \\
\hline \multicolumn{2}{|c|}{$\begin{array}{l}\text { *En } 135 \text { pacientes se realizó el proceso de búsqueda de hong } \\
\text { y anaerobios estrictos mediante tinción de Gram. }\end{array}$} \\
\hline
\end{tabular}

Los participantes de este estudio se caracterizaron clínicamente por ser hombres adultos, con al menos una co-morbilidad, diagnosticados al ingreso hospitalario con una IIAc-AC. Predominó en este grupo de pacientes la apendicitis, una IIAc-AC moderada, y como tratamiento empírico inicial ampicilina/sulbactam o ertapenem. Este perfil clínico fue similar al descrito por Dalfino y cols., en un estudio multicéntrico italiano en pacientes con 


\begin{tabular}{|c|c|c|c|c|c|c|c|c|c|c|c|c|c|c|c|c|c|c|c|}
\hline Microorganismo & $\mathbf{n}$ & AMK & SAM & ATM & FEP & CSL & CTX & CAZ & CRO & CIP & DOR & ETP & GEN & IPM & MER & TZP & POL & TGC & FOX \\
\hline E. coli & 100 & $\begin{array}{l}96 / 96 \\
(100)\end{array}$ & $\begin{array}{l}65 / 99 \\
(65,7)\end{array}$ & $\begin{array}{l}26 / 28 \\
(92,9)\end{array}$ & $\begin{array}{l}93 / 97 \\
(95,9)\end{array}$ & $\begin{array}{c}3 / 3 \\
(100)\end{array}$ & $\begin{array}{l}10 / 10 \\
(100)\end{array}$ & $\begin{array}{l}54 / 58 \\
(93,1)\end{array}$ & $\begin{array}{l}54 / 58 \\
(93,1)\end{array}$ & $\begin{array}{c}84 / 100 \\
(84,0)\end{array}$ & $\begin{array}{l}57 / 57 \\
(100)\end{array}$ & $\begin{array}{l}64 / 64 \\
(100)\end{array}$ & $\begin{array}{c}80 / 98 \\
(81,63)\end{array}$ & $\begin{array}{l}62 / 62 \\
(100)\end{array}$ & $\begin{array}{l}60 / 60 \\
(100)\end{array}$ & $\begin{array}{l}43 / 96 \\
(44,8)\end{array}$ & $\begin{array}{c}1 / 1 \\
(100)\end{array}$ & $\begin{array}{l}60 / 60 \\
(100)\end{array}$ & $\begin{array}{c}1 / 3 \\
(33,3)\end{array}$ \\
\hline E. coli no BLEE & 94 & $\begin{array}{l}90 / 90 \\
(100)\end{array}$ & $\begin{array}{l}62 / 93 \\
(66,6)\end{array}$ & $\begin{array}{l}26 / 26 \\
(100)\end{array}$ & $\begin{array}{l}92 / 93 \\
(98,9)\end{array}$ & $\begin{array}{c}3 / 3 \\
(100)\end{array}$ & $\begin{array}{l}10 / 10 \\
(100)\end{array}$ & $\begin{array}{l}54 / 54 \\
(100)\end{array}$ & $\begin{array}{l}54 / 54 \\
(100)\end{array}$ & $\begin{array}{l}80 / 94 \\
(85,1)\end{array}$ & $\begin{array}{l}55 / 55 \\
(100)\end{array}$ & $\begin{array}{l}58 / 58 \\
(100)\end{array}$ & $\begin{array}{l}77 / 92 \\
(83,6)\end{array}$ & $\begin{array}{l}58 / 58 \\
(100)\end{array}$ & $\begin{array}{l}56 / 56 \\
(100)\end{array}$ & $\begin{array}{l}40 / 93 \\
(43,0)\end{array}$ & $\begin{array}{c}1 / 1 \\
(100)\end{array}$ & $\begin{array}{l}56 / 56 \\
(100)\end{array}$ & $\begin{array}{c}1 / 1 \\
(100)\end{array}$ \\
\hline E. coli BLEE + & 6 & $\begin{array}{c}6 / 6 \\
(100)\end{array}$ & $\begin{array}{l}3 / 6 \\
(50)\end{array}$ & $\begin{array}{l}0 / 2 \\
(0)\end{array}$ & $\begin{array}{l}1 / 4 \\
(25)\end{array}$ & - & $\begin{array}{c}1 / 1 \\
(100)\end{array}$ & $\begin{array}{l}0 / 4 \\
(0)\end{array}$ & $\begin{array}{l}0 / 4 \\
(0)\end{array}$ & $\begin{array}{c}4 / 6 \\
(54,1)\end{array}$ & $\begin{array}{c}2 / 2 \\
(100)\end{array}$ & $\begin{array}{c}6 / 6 \\
(100)\end{array}$ & $\begin{array}{l}3 / 6 \\
(50)\end{array}$ & $\begin{array}{c}4 / 4 \\
(100)\end{array}$ & $\begin{array}{c}4 / 4 \\
(100)\end{array}$ & $\begin{array}{c}3 / 3 \\
(100)\end{array}$ & - & $\begin{array}{c}4 / 4 \\
(100)\end{array}$ & $\begin{array}{l}0 / 2 \\
(0)\end{array}$ \\
\hline K. pneumoniae & 14 & $\begin{array}{l}13 / 14 \\
(92,8)\end{array}$ & $\begin{array}{l}11 / 13 \\
(84,6)\end{array}$ & $\begin{array}{c}2 / 2 \\
(100)\end{array}$ & $\begin{array}{l}12 / 12 \\
(100)\end{array}$ & & $\begin{array}{l}1 / 2 \\
(50)\end{array}$ & $\begin{array}{c}5 / 5 \\
(100)\end{array}$ & $\begin{array}{c}4 / 4 \\
(100)\end{array}$ & $\begin{array}{l}13 / 14 \\
(92,8)\end{array}$ & $\begin{array}{c}6 / 6 \\
(100)\end{array}$ & $\begin{array}{c}7 / 8 \\
(87,5)\end{array}$ & $\begin{array}{l}13 / 13 \\
(100)\end{array}$ & $\begin{array}{c}7 / 8 \\
(87,5)\end{array}$ & $\begin{array}{c}7 / 8 \\
(87,5)\end{array}$ & $\begin{array}{c}6 / 6 \\
(100)\end{array}$ & - & $\begin{array}{c}6 / 6 \\
(100)\end{array}$ & - \\
\hline
\end{tabular}

IIAc-AC en quienes, al igual que en nuestro estudio, los pacientes en su mayoría eran hombres, intervenidos quirúrgicamente por apendicitis, que recibieron ampicilina/sulbactam como terapia de primera línea; y tuvieron aislamiento de al menos un microorganismo en $70,8 \%{ }^{12}$.

La mortalidad observada en nuestro estudio en los pacientes con IIAc-AC fue 15,1\% y, en más de la mitad, fue atribuible a la infección. Aunque otros autores han reportado tasas más bajas de mortalidad de $5,7^{13}$ y $6 \%{ }^{12}$. Jean y cols. ${ }^{14}$, observaron una mortalidad similar de $11,4 \%$ en un estudio multicéntrico que incluyó pacientes colombianos y en la mitad de ellos fue atribuible a la infección.

Nuestro estudio muestra que los principales microorganismos encontrados en las IIAc-AC fueron E. coli y $K$. pneumoniae, dos de las especies gramnegativas que lideran los aislados microbiológicos en los reportes internacionales de vigilancia etiológica de la IIA y en estudios multicéntricos ${ }^{6,14}$.

La comparación de la prevalencia de aislados puede variar según el denominador utilizado para su estimación. En el reporte de Hawser y cols. ${ }^{6}$, y Jean y cols. ${ }^{14}$, los aislados para E. coli y K. pneumoniae fueron 54-14 y 73,3-26,7\%, respectivamente. En nuestro estudio, una estimación idéntica mostró que 73,5 y 10,3\% de los aislados corresponden a E. coli y K. pneumoniae; sin embargo, observamos un menor valor cuando la estimación se realizó sobre el total de pacientes con IIAc-AC, 52,3 y 7,3\% de E. coli y $K$. pneumoniae, respectivamente.

La prevalencia de E. coli productora de BLEE encontrada en nuestro estudio fue de $6 \%$ y no la hubo en el caso de $K$. pneumoniae. Lo reportado por Hawser y cols. ${ }^{6}$, muestra que en IIA las especies gramnegativas predominantes fueron E. coli, K. pneumoniae y P. aeruginosa; este último hallazgo es diferente a nuestra experiencia, no siendo $P$. aeruginosa un microorganismo frecuente.
Este mismo grupo de investigadores encontró en pacientes con IIAc-AC una mayor resistencia antimicrobiana que la observada por nosotros: sensiblidad de E. coli a ampicilina/sulbactam de $55 \%$, a ciprofloxacina de $29 \%$, y a ceftriaxona de $19 \%$. En el caso de K. pneumoniae, reportaron una resistencia a ampicilina/sulbactam de $31 \%$, a ciprofloxacina de $18 \%$, y a ceftriaxona de $19 \%$. Por el contrario, en nuestro estudio observamos una mayor resistencia a carbapenémicos, posiblemente por un mecanismo asociado a producción de carbapenemasas, que ha sido identificado en diferentes hospitales colombianos $^{15-17}$. Esta diferencia puede ser explicada por la definición de caso que se utilizó en el programa de vigilancia SMART, donde sólo se utilizó la estancia hospitalaria menor a $48 \mathrm{~h}$ como un criterio de tiempo para definir IIAc-AC; en contraste a la definición clínica que usamos en nuestro estudio definida como una infección peritoneal acompañada de inflamación y/o perforación del tracto gastrointestinal o víscera hueca ${ }^{2}$ que inicia en la comunidad y/o se encontraba en desarrollo al momento del ingreso a los servicios de salud y que no cumple más de tres noches en la institución de salud ${ }^{7,11}$. Otro factor que puede influir en la diferencia encontrada es que la muestra para procesamiento microbiológico en nuestro estudio parte directamente de la definición clínica de un paciente con IIAc-AC y en los estudios el paciente es identificado a partir de un reporte del laboratorio de microbiología $a^{4-6,18}$.

El grupo microbiano grampositivo más frecuentemente aislado en nuestra casuística con IIAc-AC fue de $S$. grupo viridans, coincidiendo con la gran mayoría de los reportes de estudios microbiológicos de IIAc- $\mathrm{AC}^{7,8,19}$; reportado como completamente susceptible a los antimicrobianos probados en las cepas evaluadas.

Los estudios microbiológicos de las IIA tienen como uno de sus objetivos conocer los microorganismos más 
frecuentemente aislados y sus perfiles de susceptibilidad antimicrobiana para seleccionar el tratamiento empírico más adecuado. De acuerdo con nuestros hallazgos, adherimos a Solomkin y cols. ${ }^{7}$, en no recomendar el uso empírico de ampicilina/sulbactam para las IIAc-AC por la alta posibilidad de fallo terapéutico, dada la elevada resistencia in vitro observada. Creemos que se debe usar con precaución las quinolonas en pacientes de alto riesgo debido a la resistencia que observamos a ciprofloxacina en $E$. coli $(16 \%)$. El uso de aminoglucósidos es factible pero reconociendo que existen opciones antimicrobianas menos tóxicas, y considerando la administración diaria individualizada de los aminoglucósidos según la masa corporal magra y el volumen del líquido extracelular estimada para los pacientes que reciben estos agentes. El uso de ertapenem es una opción terapéutica teniendo en cuenta el riesgo-beneficio para minimizar el daño colateral de la presión de selección de resistencia bacteriana como lo reportaron DiNubile y cols., en los pacientes con IIA tratados con ceftrixona ${ }^{20}$.

Los carbapenémicos son una buena opción de tratamiento siempre y cuando se tenga en cuenta no cubrir innecesariamente $P$. aeruginosa, excepto en los pacientes con shock séptico como lo recomiendan Solomkin y cols. ${ }^{7}$. Según nuestros resultados y estudios previos efectuados en Colombia, se debe considerar la realización de pruebas confirmatorias para carbapenemasas en $K$. pneumoniae y así ajustar la cobertura antimicrobiana. La baja susceptibilidad a piperacilina/tazobactam en $E$. coli $(44,8 \%)$ no hace seguro su uso empírico. Tigeciclina en una opción viable in vitro para el tratamiento de las IIAc-AC por su perfil de susceptibilidad según nuestros resultados, pero debe tenerse en cuenta las limitaciones de su uso frente a la gravedad del compromiso del paciente, la posibilidad de bacteriemia simultánea y las consideraciones del uso de un derivado de tetraciclinas.

Consideramos como una limitación importante del estudio la heterogeneidad en los antimicrobianos en que se evaluó la susceptibilidad antimicrobiana, aun cuando todos los hospitales usaron métodos automatizados. La inclusión de cuatro hospitales colombianos de diferente complejidad, uno privado de cuarto nivel y tres públicos de tercer nivel, de cuatro ciudades diferentes, refleja en parte la situación de la IIAc-AC en Colombia, dado que el estudio no incluyó un número mayor de hospitales en otras ciudades capitales del país.
En conclusión, los principales microorganismos a cubrir en una terapia antimicrobiana empírica en pacientes con IIAc-AC son E. coli, K. pneumoniae y $S$. grupo viridans. Es necesario conocer la susceptibilidad antimicrobiana en cada región para seleccionar un tratamiento empírico adecuado a cada institución.

Agradecimientos. A los Centros de Investigación del Hospital General de Medellín, Hospital Universitario San Jorge de Pereira, Hospital Universitario Hernando Moncaleano Perdomo de Neiva, Fundación Santa Fe de Bogotá. A Elsa de la Cadena del Centro Internacional de Entrenamiento e Investigaciones Médicas (CIDEIM) en Cali. A Sergio Cifuentes del Hospital Universitario San Jorge de Pereira.

\section{Resumen}

Introducción: La infección intra-abdominal complicada adquirida en la comunidad (IIAc-AC) es una causa frecuente de abdomen agudo. Objetivo: Identificar el perfil clínico y microbiológico de la IIAc-AC en cuatro hospitales de Colombia. Métodos: Estudio descriptivo, prospectivo entre 07-2012 y 09-2014 en pacientes de 15 o más años con IIAc-AC. Se midieron las frecuencias de variables socio-demográficas, clínicas, diagnóstico, aislamientos y susceptibilidad antimicrobiana del primer cultivo obtenido asépticamente del sitio de infección. Resultados: 192 pacientes incluidos, 62\% hombres, edad media 47,3 años; 38,4\% con co-morbilidad, 13\% hospitalizados en el último año y $9,4 \%$ recibieron antimicrobianos en los últimos seis meses. Fueron admitidos 44,3\%, por apendicitis $17,7 \%$ por peritonitis y $16,7 \%$ por perforación intestinal. El $64,1 \%$ de las IIAc-AC fue moderada y tratada con ampicilina/sulbactam (SAM) y ertapenem. En 70,8\% se aisló al menos un microorganismo en: $65,1 \%$ bacilos gramnegativos (80,0\% Escherichia coli, 44,8\% susceptible a piperacilina/tazobactam, $65,7 \%$ a SAM y $11,2 \%$ Klebsiella pneumoniae, $85 \%$ susceptibles a SAM) y en $16,7 \%$ especies grampositivas $(28,1 \%$ Streptococcus grupo viridans). La mediana de hospitalización fue siete días y 15,1\% fallecieron. Conclusión: Escherichia coli y $K$. pneumoniae en IIAc-AC son los principales microorganismos a cubrir en la terapia empírica y es necesario conocer la susceptibilidad antimicrobiana en cada región para seleccionar un tratamiento empírico adecuado.

\section{Referencias bibliográficas}

1.- Fenyo G, Boijsen M, Enochsson L, Goldinger M, Grondal S, Lundquist P, et al. Acute abdomen calls for considerable care resources. Analysis of 3727 in-patients in the county of Stockholm during the first quarter of 1995. Lakartidningen 2000; 97: 4008-12.

2.- Mazuski J E, Solomkin J S. Intra-abdominal infections. Surg Clin North Am 2009; 89: 421-37.

3.- Montravers P, Chalfine A, Gauzit R,
Lepape A, Pierre Marmuse J, Vouillot C, et al. Clinical and therapeutic features of non postoperative nosocomial intra-abdominal infections. Ann Surg 2004; 239: 409-16.

4.- Villegas M V, Blanco M G, Sifuentes-Osornio J, Rossi F. Increasing prevalence of extended- 
spectrum-beta-lactamase among Gram-negative bacilli in Latin America-2008 update from the Study for Monitoring Antimicrobial Resistance Trends (SMART). Braz J Infect Dis 2011; 15: 34-9.

5.- Salles M J, Zurita J, Mejía C, Villegas M V Latin America Working Group on Bacterial Resistance. Resistant gram-negative infections in the outpatient setting in Latin America. Epidemiol Infect 2013; 141: 2459-72.

6.- Hawser S, Hoban D J, Badal R E, Bouchillon S K, Biedenbach D, Hackel M, et al. Epidemiology and antimicrobial susceptibility of Gram-negative aerobic bacteria causing intra-abdominal infections during 2010-2011. J Chemother 2014: 1973947814 Y0000000164.

7.- Solomkin J S, Mazuski J E, Bradley J S, Rodvold K A, Goldstein E J, Baron E J, et al. Diagnosis and management of complicated intra-abdominal infection in adults and children: Guidelines by the Surgical Infection Society and the Infectious Diseases Society of America. Clin Infect Dis 2010; 50: 133-64.

8.- Herzog T, Chromik A M, Uhl W. Treatment of complicated intra-abdominal infections in the era of multi-drug resistant bacteria. Eur J Med Res 2010; 15: 525-32.

9.- Membrilla-Fernández E, Sancho-Insenser J J, Girvent-Montllor M, Álvarez-Lerma F, Sitges-Serra A. Secondary Peritonitis Spanish Study Group. Effect of initial empiric antibiotic therapy combined with control of the infection focus on the prognosis of patients with secondary peritonitis. Surg Infect (Larchmt) 2014; 15 (6): 806-14.

10.- Chong Y P, Bae I G, Lee S R, Chung J W, Jun $\mathrm{J} \mathrm{B}$, Choo E J, et al. Clinical and economic consequences of failure of initial antibiotic therapy for patients with community-onset complicated intra-abdominal infections. PLoS One 2015; 10 (4): e0119956.

11.- Cohen A L, Calfee D, Fridkin S K, Huang S S, Jernigan J A, Lautenbach E, et al. Recommendations for metrics for multidrugresistant organisms in healthcare settings: SHEA/HICPAC Position paper. Infect Control Hosp Epidemiol 2008; 29: 901-13.

12.- Dalfino L, Bruno F, Colizza S, Concia E, Novelli A, Rebecchi F, et al. Cost of care and antibiotic prescribing attitudes for communityacquired complicated intra-abdominal infections in Italy: a retrospective study. World J Emerg Surg 2014; 9: 39

13.- Sturkenboom M C, Goettsch W G, Picelli $\mathrm{G}$, in 't Veld B, Yin D D, de Jong R B, et al. Inappropriate initial treatment of secondary intra-abdominal infections leads to increased risk of clinical failure and costs. Br J Clin Pharmacol 2005; 60: 438-43.

14.- Jean S S, Ko W C, Xie Y, Pawar V, Zhang D, Prajapati G, et al. Clinical characteristics of patients with community-acquired complicated intra-abdominal infections: a prospective, multicentre, observational study. Int J Antimicrob Agents 2014; 44: 222-8.

15.- Mojica M F, Correa A, Vargas D A, Maya J J,
Montealegre M C, Rojas L J, et al. Colombian Nosocomial Bacterial Resistance Study Group. Molecular correlates of the spread of KPCproducing Enterobacteriaceae in Colombia. Int J Antimicrob Agents 2012; 40: 277-9.

16.- Cuervo S I, Sánchez R, Gómez-Rincón J C, Almenares C, Osorio J P, Vargas M J. Behavior of carbapenemase-producing Klebsiella pneumoniae cases in cancer patients at a third level hospital in Bogotá, D.C. Biomedica 2014; 34: 170-80.

17.- Pacheco R, Osorio L, Correa A M, Villegas M V. Prevalence of gram-negative bacteria harboring bla KPC gene in Colombian hospitals. Biomedica 2014; 34: 81-90.

18.- Hawser S P, Badal R E, Bouchillon S K, Hoban D J, Biedenbach D J, Cantón R, et al. Monitoring the global in vitro activity of ertapenem against Escherichia coli from intra-abdominal infections: SMART 2002-2010. Int J Antimicrob Agents 2013; 41: 224-8.

19.- Blot S, De Waele J J, Vogelaers D. Essentials for selecting antimicrobial therapy for intra-abdominal infections. Drugs 2012; 72: e17-32.

20.- Dinubile M J, Friedland I, Chan C Y, Motyl M R, Giezek H, Shivaprakash M, et al. Bowel colonization with resistant gramnegative bacilli after antimicrobial therapy of intra-abdominal infections: observations from two randomized comparative clinical trials of ertapenem therapy. Eur J Clin Microbiol Infect Dis 2005; 24: 443-9. 